\title{
Die Fauna von Hunas (Nördliche Frankenalb) im Rahmen der deutschen Quartärfaunen
}

\author{
Von Florian Heller, Erlangen
}

Mit 1 Abbildung

$\mathrm{Z}$ u s a m m e n f a s su $\mathrm{ng}$. Nach der vorläufigen Einstellung der sich über 9 Jahre erstreckenden Ausgrabungen wird nunmehr eine erste detailliertere Übersicht über die Zusammensetzung der Fauna aus den einzelnen Schichten des insgesamt mit 22,5-23 m Mächtigkeit ermittelten Quartärprofils der Höhlenruine von Hunas (Nördliche Frankenalb) gegeben. Für die Klärung der Altersfrage der gesamten Ablagerungen sind insbesondere die Reste der Kleinsäuger von Wichtigkeit, die sich stellenweise in großer Menge fanden. Unter den Formen, die zu einem guten Teil mit altquartären Arten in Verbindung gebracht werden müssen, bedeuten Allocricetus bursae ScHAUв und Parapodemus coronensis Schaub sicherlich eine regelrechte Uberraschung. Bestimmt jünger als die Fauna von Steinheim a. d. Murr, dürfte die Fauna von Hunas im wesentlichen der RißEiszeit mit ihren verschiedenen Abschnitten, sowie dem Riß-Würm-Interglazial angehören.

$\mathrm{Sum} \mathrm{mary}$. After nine years working the excavations of the cave-ruines of Hunas (East of Nuremberg, Bavaria) were interrupted for some time. Now a more detailed account is given of the faunas in the several strata of the Quaternary-profile having a thickness between 22,5 and $23 \mathrm{~m}$. For the determination of the age of the deposits the abundantly found remains of small mammals are of high importance. Among the forms which in many cases must be brought into connexion with old quaternary species, Allocricetus bursae Schaub and Parapodemus coronensis Schaub are extremely surprising. Surely being younger than the fauna of Steinheim a. d. Murr, the fauna of Hunas should essentially belong to the Riss glaciation with its different phases and also to the Riss-Würm-Interglacial.

Wer von den Teilnehmern der Deuqua-Tagung 1962 in Nürnberg heute die Höhlenruine Hunas bei Hartmannshof/Ldkr. Hersbruck besuchen würde, müßte wohl erneut über das in weiterer, zweijähriger Grabungsarbeit Erreichte staunen. Von der damaligen Grabungssohle ausgehend, wurden zunächst noch einige Querprofile im Abstand von $1 \mathrm{zu} 1 \mathrm{~m}$ (insgesamt sind es nunmehr 14) mit einer maximalen Schichtmächtigkeit von nahezu $10 \mathrm{~m}$ erschlossen. Das dadurch entstandene große Längsprofil (Abb. 1) zeigt aufs beste den genauen Schichtverlauf: das bergwärtige Einfallen der Verfüllungen, sowie deren Zusammensetzung aus mehreren, miteinander verknüpften Schuttkegeln. Zum Abschluß unserer vorläufig letzten Grabungskampagne'wurde schließlich der schon früher in Angriff genommene breite Schlitz, der durch die mächtigen Gesteinsblöcke der tieferen Versturzmassen hindurch den einstigen natürlichen Höhlenboden erreichen sollte, vorangetrieben. Nun erst zeigte sich in vollem Umfange, welch gewaltige Mengen an Schuttmaterial der verschiedensten Größenkategorien den riesigen, im Laufe von Jahrtausenden eingestürzten Hohlraum erfüllen. Der gewachsene Fels des Malmdolomits wurde erst in etwa $12^{1 / 2} \mathrm{~m}$ Tiefe unter einer Schicht von rosa bis rot gefärbten, eingeschlämmten Kreidetonen angetroffen, woraus sich eine Mindestmächtigkeit der gesamten Verfüllungsmasse von rund $22,5-23 \mathrm{~m}$ errechnet.

Aus den gelegentlich zwischen den großen Versturzblöcken auftretenden Partien feinkörnigeren Sedimentmaterials kamen, wie eigentlich erwartet, kaum mehr Reste von Wirbeltieren zum Vorschein, ein Beweis dafür, daß die „Hunaser Höhle“ während der Anfangsphase ihres Zubruchgehens noch keine nennenswerten Verbindungen zur Außenwelt besaß. Soweit es sich um genauer bestimmbare faunistische Fundstücke handelt, werden diese als die ältesten aus dem gesamten Schichtenkomplex natürlich besondere Beachtung verdienen.

Die Ausbeute an paläontologischem und urgeschichtlichem Material aus der bereits bekannten oberen Abfolge des Profils (Schichten D-M) war auch in den beiden letzten

8 Eiszelt und Gegenwart 


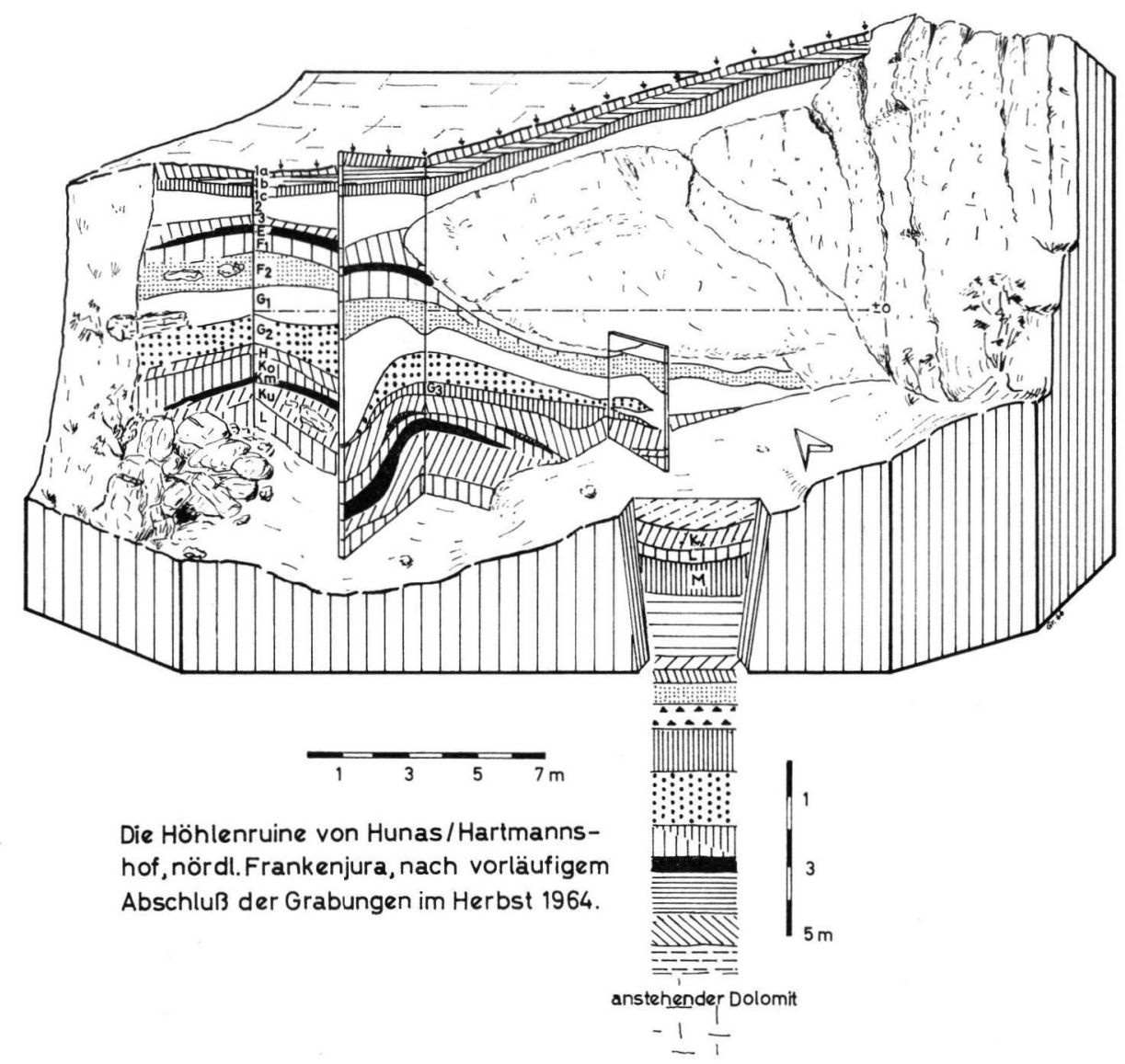

Abb. 1. Das Quartär der Höhlenruine von Hunas/Hartmannshof, nördl. Frankenjura.

$1 \mathrm{a}-\mathrm{c}, 2,3=\mathrm{Gehängeschutt.} \mathrm{Darunter} \mathrm{folgt} \mathrm{(nur} \mathrm{lokal} \mathrm{vertreten} \mathrm{und} \mathrm{in} \mathrm{der} \mathrm{Abb.} \mathrm{nicht} \mathrm{dargestellt)}$ kaltzeitlicher Schutt. E, F = Interglazialsedimente. G, H, K, L, M = kaltzeitlicher Schutt (vorwiegend Frost- und Blockschutt). - Unter $M$ folgt in einem Tiefschnitt, der zur Feststellung der Gesamtmächtigkeit der Höhlenverfüllungen bis zum anstehenden Dolomit - dem alten HöhlenBoden - niedergebracht wurde, Blockschutt und angeschwemmtes Material (oben zum Teil stark versintert). Diese zumeist fundleeren Ablagerungen sind noch nicht genauer untersucht. Die kleinen Pfeile entlang der Landoberfläche deuten die einzelnen Grabungsabschnitte an. - Der große Richtungspfeil weist nach Westen.

Jahren überaus beachtlich. Von besonderem Interesse dürfte sein, daß mit Annäherung an den offensichtlich seinerzeitigen Haupt-Höhleneingang sich immer zahlreichere Kleinwirbeltierreste (Mikrofauna) einstellten. Recht auffällig waren dabei in einigen Fundniveaus fast ganz aus Froschknochen bestehende Vorkommen. In gewissen Schichten (G, sowie J und K) fanden sich nicht nur nesterartige Anhäufungen von Kleinsäuger-, Fisch-, Amphibien-, Reptil- und Vogel-Resten, sondern stellenweise in mehrfacher Folge übereinander, regelrechte ausgedehnte Knochenlager. Ihr Zustandekommen erklärt sich aus der Akkumulation unzähliger Gewölle von Raubvögeln, die einstmals in den Nischen am Höhleneingang nisteten oder diese eigens zum Zweck der ungestörten Abgabe ihres unverdaulichen Kropfinhaltes aufsuchten. Es wird außerdem bewiesen durch noch vollkommen in der ursprünglichen Form erhalten gebliebene einzelne fossile Gewölle. Dem Magen erbeuteter Hühnervögel und anderer Körnerfresser entstammen die zahlreich ge- 
fundenen, oftmals schön gerundeten und wie poliert aussehenden Kieselsteinchen, aber auch verschiedene Bruchstücke von Jura-Belemniten, welche als ortsfremd aufgepickt bezeichnet werden müssen, da sie unmöglich aus dem anstehenden Dolomit der Höhlenruine von Hunas ausgewittert sein können.

Paläosoziologisch liegt in Hunas somit der keineswegs sehr häufige Fall vor, daß die Gesamtfauna des Fundplatzes sich nicht auf einen ganz bestimmten zönotischen Typ beschränkt, sondern dem Zusammenwirken verschiedener ökologischer, bio- und thanatozönotischer Faktoren zu verdanken ist. Mit ihrem Schwerpunkt stellt sie, jedenfalls in bestimmten Bereichen des Schichtprofils, ganz besonders aber mehr im Innern der ehemaligen Höhle, eine Bären-Fauna im Sinne Kretzor's dar. Reste von Hyänen wurden nach wie vor nicht gefunden; Löwe und Wolf sind nur mit spärlichen Belegen vertreten. Die Beimengung von Knochenresten mittelgroßer bis großer Pflanzenfresser, wie Reh, Hirsch, Elch, Pferd, Nashorn und schließlich Boviden, beruht zu einem Teil zweifellos auf völlig natürlichen Vorgängen, indem es sich hierbei um mit dem Gesteinsschutt in die Höhle hineingeratene Skelettelemente verendeter Tiere handelt. Ein weiterer, jedoch unbedeutender Teil dürfte von Raubtieren eingeschleppt worden sein. In jenen Fundschichten aber, in welchen die mehr oder weniger häufig angetroffenen Artefakte, Feuerstellen oder sonstigen Holzkohlenanhäufungen die Anwesenheit des steinzeitlichen Menschen verraten, handelt es sich bei den Knochenresten sicherlich auch um dessen Jagdbeute, bzw. Überbleibsel seiner Mahlzeiten.

Die bereits erwähnten Mikrofauna-Anhäufungen (Raubvogel-Faunen, wiederum im Sinne KRETzoI's) sind im wesentlichen ein Charakteristikum der kleinstückigen Bruchschuttschichten nahe oder unmittelbar am ehemaligen Höhleneingang. Ihre nach einzelnen Horizonten verschiedenartige Zusammensetzung ist für die klimatologische und chronologische Ausdeutung des Gesamtprofils von Hunas von ausschlaggebender Bedeutung.

Die fast ausschließliche „Höhlenbären-Fauna “ in den Schichten E und F mit den hauptsächlichsten Begleitformen Reh, Hirsch und Biber, jedoch ohne eigentliche glaziale oder Steppenelemente, wie z. B. Ren usw., repräsentiert eine typische Waldfauna mit der notwendigen Einweisung in ein Interglazial. Demgegenüber wird der schon auf rein petrographisch-pedologischer Basis ermittelte glaziale Charakter des unterlagernden Bruchschuttkomplexes $G$ unterstrichen durch die im Vergleich zu den Großsäugerformen unbedingt aussagefähigeren Elemente der Mikromammalier-Fauna. Letztere trägt stark ausgeprägten Steppencharakter, wie die vorherrschenden Wühlmäuse, unter ihnen zwar nicht allzu häufig Lemmus lemmus L. und ganz vereinzelt Dicrostonyx beweisen. Dazu gesellen sich eine Ochotona-Art, ein großer Cricetus cricetus, Steppenhamster, eine TalpaArt, Vertreter des Sorex araneus-Formenkreises, eine kleine Neomys-Art, während die südlichen Bereichen zugehörige Gattung Crocidura vollkommen fehlt. An kleinen Raubtieren zu erwähnen wären ferner verschiedene Musteliden. Recht gut zu dieser Faunengesellschaft passen schließlich einige wenige Reste von Ren.

Leider liegen aus der petrographisch recht charakteristischen Schicht $\mathrm{H}$, die schon auf Grund ihres Pollendiagrammes nur ein Interstadial repräsentieren kann, relativ wenige Kleinsäugerreste vor, was hinsichtlich weiterer Aussagen zu einer gewissen Vorsicht zwingt. Reichlich finden sich wiederum Kleinwirbeltierreste in der Lehmschicht I und dem darunter folgenden Bruchschutt $\mathrm{K}$, doch lassen sich die einzelnen Faunen bedauerlicherweise nur in Ausnahmefällen auseinanderhalten. Durch die Tatsache, daß der offensichtlich noch nach seiner Ablagerung sehr lose gepackte Bruchschutt allenthalben Zwischenräume aufwies, dürften bereits der jüngeren Lehmschicht zugehörige Faunenelemente zunächst tiefer gesackt sein, wodurch eine Vermischung eintrat. Insgesamt gesehen ergeben sich bei der Durchmusterung des betreffenden Fossilmaterials trotz Anwesenheit der 
meisten bereits oben angeführten Kleinsäugerarten doch auch gewisse Unterschiede. Unter den Wühlmäusen sind nämlich auffallend viele Reste einer Cletbrionomys-Art vorhanden, außerdem nicht gerade selten solche einer kleinen Muriden-Art, welch beide zumindest auf einen etwas stärkeren Baumbestand bzw. eine gewisse Bewaldung der Landschaft hinweisen.

Die geschilderten paläontologischen Befunde am Quartärprofil von Hunas, das über Schichtgliedern mit mehrmaligem Auftreten von Steppenfaunen, wenn auch unterschiedlicher Prägung, eine mehr oder weniger ausgesprochene Höhlenbären-Fauna interglazialen Charakters zeigt, dürften für mitteleuropäische Verhältnisse zweifellos etwas Besonderes darstellen. Jedenfalls stehen sie nach unseren Erfahrungen in absolutem Gegensatz zu den üblichen Abfolgen in jungquartären Höhlenablagerungen, die bekanntlich kaum über das Frühwürm zurückreichen. Die entsprechenden Beweise für höheres Alter, und dies sei gegenüber allenfallsigen Einwänden noch einmal ganz besonders hervorgehoben, sind zunächst in dem ungewöhnlichen Erhaltungszustand, d.h. der stärkeren Verwitterung bzw. Zersetzung aller größeren Knochenreste zu erblicken, wodurch es fast nie gelingt, selbst die kompaktesten Skelettelemente z. B. des Fußes und der Hand völlig intakt $\mathrm{zu}$ bergen. Wiederholt wurde vom Verf. in Wort und Schrift aber auch bereits auf die größere Primitivität der mit einem reichhaltigen Material vorliegenden Reste an Höhlenbären hingewiesen.

Ausschlaggebend für die Beurteilung des Alters der Gesamtfauna erscheinen jedoch die Ergebnisse der Artenbestimmung, die nach Auffindung der reichen Mikrofaunen-Reste besonders vorangetrieben wurde. Im Vordergrund des Interesses steht hier die allgemeine phylogenetische Entwicklungshöhe der Wühlmäuse, d. h. der Arvicoliden bzw. Microtinen, deren Kieferchen zu Tausenden für unsere Untersuchungen zur Verfügung standen. Daß weder Vertreter von Primitivformen wurzelzähniger Gattungen wie z. B. Promimomys, Baranomys usw., noch solche der evoluierteren Dolomys und Mimomys vorkommen, verstand sich nach Lage der Dinge eigentlich von selbst. Es fehlen aber auch die PliomysArten, die unter den jüngeren Altquartärfaunen von Deutschland jene aus der Sackdillinger Höhle (Opf.), von Erpfingen (Württ.), Hohensülzen (Rheinhessen) und selbst aus den Sanden von Mauer bei Heidelberg charakterisieren. Als einzige wurzelzähnige Wühlmäuse kommen in Hunas allein Clethrionomys-Formen vor, allerdings in z. T. offenbar noch unbekannten und vielleicht auch etwas primitiveren Arten. Beherrscht wird das "Wühlmausspektrum" ohne Frage durch die verschiedenen - auch neuen - Arten von Microtus, die oft nur sehr schwer auseinanderzuhalten sind. Dies gilt namentlich für den Microtus-arvalis-agrestis-Formenkreis, in welchem auffallend kleinwüchsige Vertreter auftauchen, die man eigentlich nur mit der Art M.arvalinus HINT. aus den jüngeren Altquartärbildungen identifizieren kann, zumal gleichzeitig auch einzelne Formen von M. nivalinus Hint. und M. ratticepoides HINT. vorkommen. Andererseits gibt es gerade von letzteren wiederum großwüchsigere Typen in allen Übergängen zum echten $M$. ratticeps KEYs. \& BlAs. (=M. oeconomus) typisch jungquartärer Ablagerungen. In stratigraphischer Hinsicht sind den genannten altertümlicheren Microtus-Arten aber auch die nicht gerade häufigen Pitymys-Formen anzuschließen, die als $P$. arvaloides Hinton und P. gregaloides, bzw. aff. gregaloides Hint. (Hohensülzen, Sackdillinger Höhle, Erpfingen) bestimmt wurden. Wesentlich charakteristischer in ihrem Habitus erscheinen die Vertreter der Gattung Arvicola. Im allgemeinen kleiner als die typischen jungquartären und rezenten, weisen sie zudem, wenn auch z. T. etwas abgemildert, dasselbe Verhalten der wechselnden Schmelzbanddicke ihrer Molaren auf, welches die ersten aus MimomysFormen hervorgegangenen altquartären Arvicola-Arten kennzeichnet. Die in Hunas geborgenen Reste erinnern in jeder Hinsicht an A. greenii HINT. (Erpfingen, Mauer, Mosbach, Pilgerhaus bei Weinheim) und A.praeceptor HINT. (Früh-Mittelterrasse der Themse). 
Ein recht eigenartiges Bild liefern hinsichtlich ihrer Größenverhältnisse auch die Spitzmäuse der Gattung Sorex. Neben einer sehr kleinen Form, wahrscheinlich S. minutus L., finden sich, zwar ziemlich selten, mittelgroße Typen, die mit S. araneoides HeLleR (Sackdillinger Höhle, Erpfingen) gut übereinstimmen; etwas häufiger ist eine Größenkategorie, die S. subaraneus HELler von Erpfingen entspricht. Die zahlreichen noch größeren Vertreter erreichen nicht nur die Maße der jungquartären und rezenten S. araneus L., sondern gehen vielfach sogar darüber hinaus. Unter den Musteliden wiederum fällt im Bau des Mandibelknochens die große Ähnlichkeit mit gewissen altquartären Vorläuferformen auf. So lassen sich insbesondere die Kieferchen in der ungefähren Größe rezenter Wiesel praktisch nicht von Mustela praenivalis Konmos (Sackdillinger Höhle, Erpfingen) unterscheiden.

Eine regelrechte Überraschung erbrachte schließlich die Bestimmung der kleinen Steppenhamster und der echten Mäuse, von denen glücklicherweise ein ausreichendes Material zur Verfügung steht. Bei ersteren handelt es sich einwandfrei nicht um den jungquartären Cricetiscus songorus PALL. foss., sondern um den altquartären Allocricetus bursae Schaub (Sackdillinger Höhle, Erpfingen). Die Muriden-Reste, die sich durch kleine Besonderheiten im Bau des Unterkiefers schon von Apodemus sylvaticus L. oder A. flavicollis MELCH. unterscheiden, repräsentieren auf Grund der charakteristischen Bewurzelung und sonstiger Merkmale ihrer Oberkiefermolaren Parapodemus coronensis SCHAub, eine Art, die bisher nur aus dem jüngeren Altquartär von Brassó (Kronstadt) in Siebenbürgen bekannt wurde. Schließlich scheinen auch die Reste von Zwergpfeifhasen nicht dem gewöhnlichen jungquartären Vertreter Ochotona pusillus PALL. anzugehören, sondern einer neuen Art.

Alles in allem dürften diese Ausführungen gezeigt haben, daß in der Mikrofauna von Hunas einwandfrei noch vereinzelt typische Elemente einer älteren, vorangegangenen Tierwelt enthalten sind, die aber mehr und mehr zurücktreten. Das Gros der Arten dagegen wird gebildet von Formen, die gewissermaßen verschiedene Phasen der Umprägung älterer Vorläufer zu jungquartären und rezenten Typen darstellen.

Damit ist bereits der Gesamtcharakter der Fauna von Hunas hinsichtlich ihrer Altersstellung umrissen. Ganz erheblich artverschieden von den Altquartärfaunen mit Mimomys (Gundersheim/Rheinhessen, Deinsdorf/Opf., Schernfeld/Mfr., sowie Hohensülzen/Rheinhessen), aber auch von jenen mit reichlich vorkommenden Pliomys-Arten (Sackdillinger Höhle, Erpfingen), bestehen selbst noch keine engeren Beziehungen zu den Faunen der Mauerer und Mosbacher Sande, sowie den entsprechenden Ablagerungen am Pilgerhaus bei Weinheim/Bergstraße. Ohne Bedenken wird man sich auch dahingehend äußern dürfen, daß die Gesamtfauna von Hunas sich sogar noch gegenüber jener von Steinheim a. d. Murr als wesentlich jünger erweisen wird. In der Hauptsache der Rißeiszeit mit ihren verschiedenen Abschnitten und dem Riß-Würm-Interglazial zugehörig, wie aus rein quartärgeologischen Gründen bereits seit längerem gefolgert wurde, füllt sie eine bisher noch immer bestehende, empfindliche stratigraphisch-faunengeschichtliche Lücke aus, deren Schließung der allgemeinen Kenntnis vom Ablaufe des Quartärs dienen wird.

Manuskr. eingeg. 19. 3. 1965

Anschrift des Verf.: Prof. Dr. Fl. Heller, 852 Erlangen, Schloßgarten 5, Geolog. Institut der Univ. 